\title{
Espaces d'espèces. Les coquillages ordinaires chez soi ou une invitation à un voyage intérieur
}

Ordinaries seashells at home or an invitation to a journey within

\section{Anne Monjaret}

\section{(2) OpenEdition}

\section{Journals}

Édition électronique

URL : https://journals.openedition.org/tc/6545

DOI : $10.4000 /$ tc. 6545

ISSN : 1952-420X

Éditeur

Éditions de l'EHESS

\section{Édition imprimée}

Date de publication : 15 décembre 2012

Pagination : 62-77

ISBN : 978-2-7351-1534-1

ISSN : 0248-6016

\section{Référence électronique}

Anne Monjaret, «Espaces d'espèces. Les coquillages ordinaires chez soi ou une invitation à un voyage intérieur », Techniques \& Culture [En ligne], 59 | 2012, mis en ligne le 15 décembre 2015, consulté le 29 septembre 2022. URL : http://journals.openedition.org/tc/6545; DOI : https://doi.org/10.4000/tc.6545 


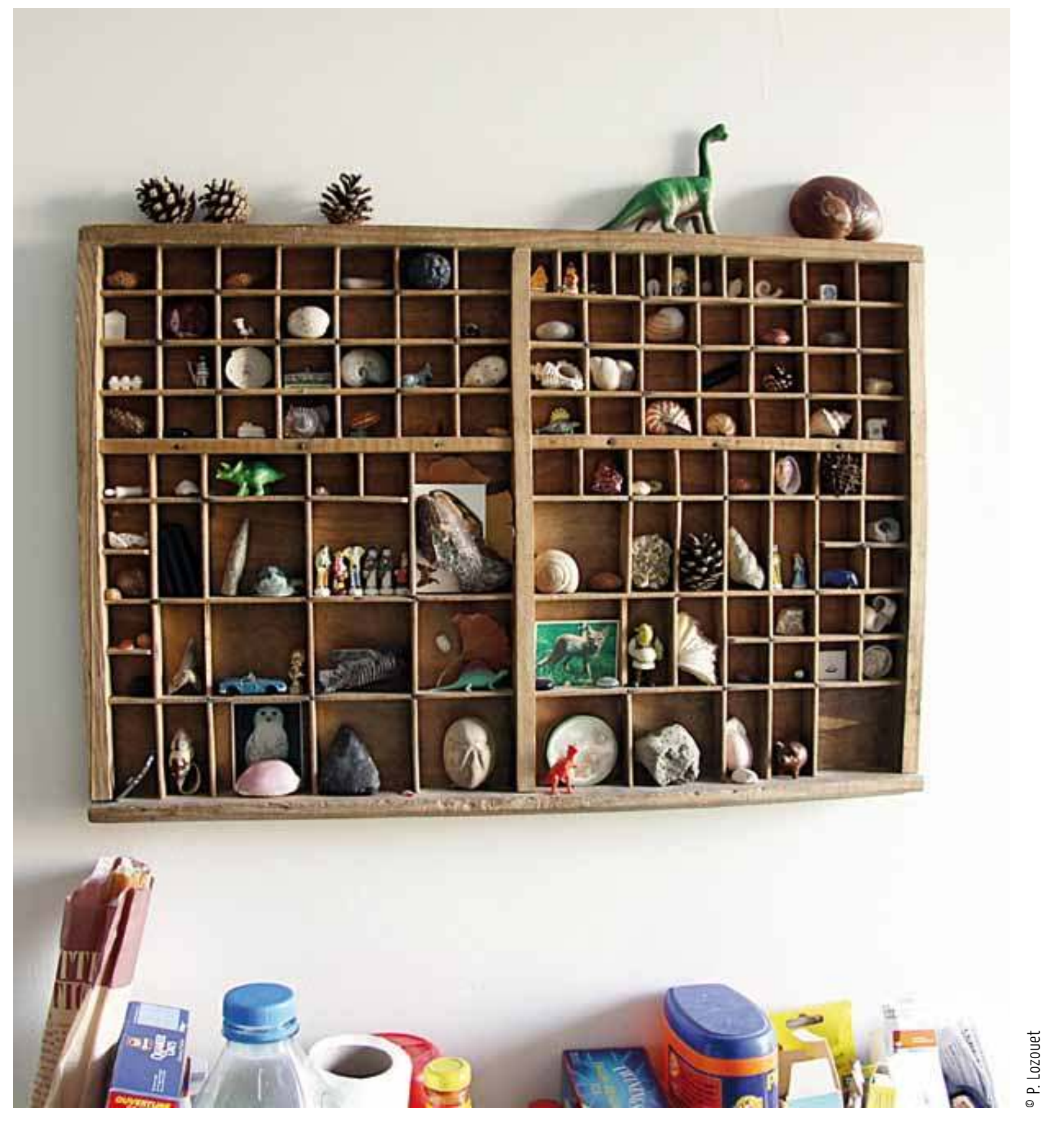




\section{ESPACES D’ESPÈCES}

\section{Les coquillages ordinaires chez soi ou une invitation à un voyage intérieur}

Les coquilles ou « coquillages »- terme entendu ici dans son acception commune - et les fossiles, prélevés de leur environnement naturel, connaissent souvent une deuxième vie qui les éloigne de leur terre d'origine ou plus précisément de leur mer d'origine.

Ils se retrouvent alors parfois dans un laboratoire scientifique où ils seront triés, scrutés, classés, conservés, parfois aussi plus modestement dans la vitrine d'une boutique où ils servent à la composition d'une décoration estivale, parfois encore dans nos intérieurs domestiques où ils rejoignent l'armée de bibelots de tout genre. C'est à cette dernière situation que nous nous intéresserons, et ce d'autant plus qu'elle n'a jamais fait l'objet d'une réflexion en soi de la part des ethnologues qui ont, par exemple, étudié les intérieurs de familles ouvrières ou plus largement populaires autour de Nantes et Paris. Les logements citadins sont cependant loin d'être dépourvus de références à la nature, mais l'« intrusion écologique » analysée par certains auteurs dans les années 1980 (Deniot, 1986; Segalen, 1987) apparaît nettement plus végétale. Quant au décor des maisons de pêcheurs décrit par Noëlle Gérôme ces mêmes années, il renvoie à la mer plutôt qu'à la plage, à l'univers maritime plutôt qu'à l'univers balnéaire. Même l'« étoile de mer ramenée d'un filet et enrubanné de rose » est là pour évoquer l'activité de pêche de son propriétaire (Gérôme, 1981 : 44).

Ainsi, nombre des travaux portant sur l'habitat pourraient laisser à penser que les coquillages n'ont pas leur place dans les intérieurs domestiques. Est-ce un effet d'enquête? Les coquillages seraient-ils passés inaperçus aux yeux des ethnologues? Ou sont-ce les familles étudiées qui ne les valorisaient pas? Pourtant, une étude plus récente vient confirmer leur présence, du moins dans des appartements d'un quartier
"Les lieux tels qu'ils existent dans la mémoire sont plus qu'un décor, ils sont une coquille. Sorte d'enveloppe première entre soi et le reste du monde. Coquille peu à peu fossilisée par le temps dont l'empreinte marque les terres et les pierres du présent comme de l'avenir.

(Muxel, $1996: 47$ ) 


\section{L'orchidée au fossile \\ Le voisinage entre une orchidée et un fossile semble étrange. Pourtant cette rencontre n'est pas fortuite: la plante qui a pris trop de volume risque la chute à tout moment si elle n'est pas stabilisée, le fossile vient là comme un joli contrepoids.}

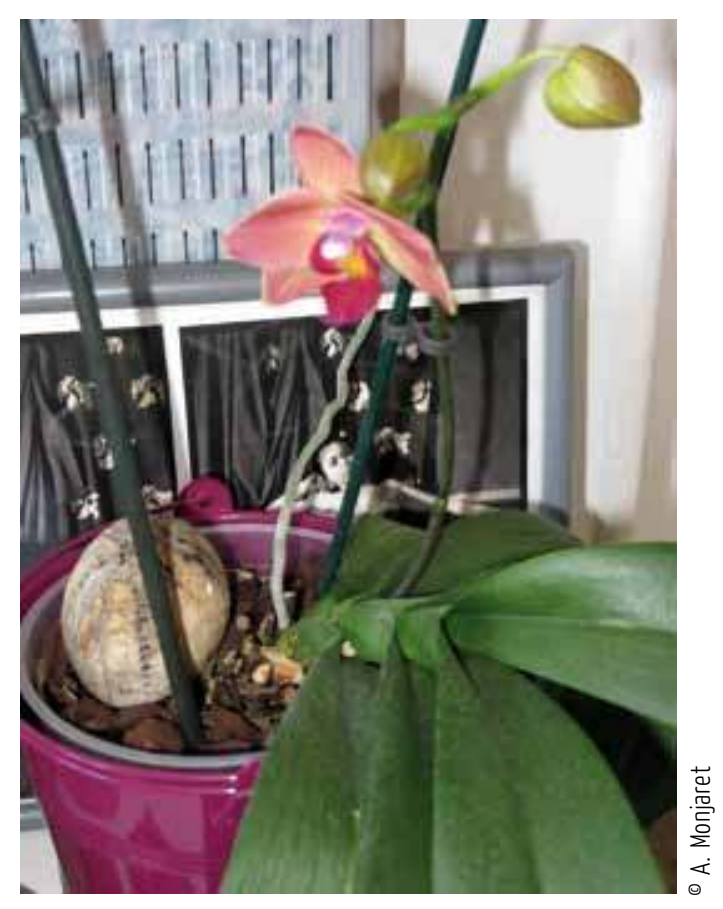

populaire de Lyon (Halitim, 1996). Est-ce parce que la plupart des habitants rencontrés ont connu une expérience de déplacement, celle d'une immigration nationale ou internationale? Est-ce aussi parce qu'ils appartiennent à une génération qui connaît les voyages et les objets qui les incarnent, qui sait ce qu'ils peuvent représenter? Ici les coquilles, à la fois précieuses et si banales, se transforment en bibelot, venant se fondre parmi les autres souvenirs. C'est cette logique d'ensemble qui retient l'attention de Nadine Halitim et non la spécificité de certaines familles d'objets. Ainsi, les coquilles ne rencontrent pas la considération qu'ils ont auprès des malacologues ou de férus collectionneurs. Dépouillées de leur aura scientifique, n’ayant rien de particulier, elles sont rendues en quelque sorte à la vie ordinaire.

À nos yeux, c'est bien ce « rien de particulier » qui fait tout l'intérêt de leur étude dans les espaces domestiques. C'est cette apparente banalité qui a aiguisé notre curiosité. Ces petits objets, souvent modestes, ont la faculté de s'immiscer dans les moindres recoins de la maison, s'incruster discrètement. Ils semblent se nicher partout, sont toujours là, tout en sachant faire oublier leur présence. Où les trouvons-nous exactement? Collection ordonnée ou joli fouillis, accumulation disséminée, quelle mise en scène leur est-elle associée? Pourquoi acceptons-nous cet envahissement sournois? Quels motifs nous poussent à ramener et accueillir chez soi ces coquilles ou fossiles, à les conserver, à les exposer jusqu'à ne plus savoir qu'en faire? Quelles fonctions et valeurs leur attribuonsnous? Autrement dit, comment expliquer l'attachement que nous leur vouons?

Pour répondre à ces questions, nous sommes allés puiser dans nos expériences personnelles, nos flâneries urbaines. Nous avons observé et interrogé assez librement, c'està-dire sans protocole serré d'enquête, nos proches et nos amis ${ }^{1}$ issus plutôt de la classe moyenne urbaine. Aucun d'eux n'est à proprement parler collectionneur de coquillages (Vincent, 2011), mais tous en possèdent. L'un a fait de sa curiosité pour ces espèces marines un métier mais la plupart se présentent comme des glaneurs occasionnels. Ils n’ont rien du passionné ordinaire (Bromberger, 1998). Ils ont accumulé deci delà, au fil des années, un trésor qu'ils mettent ou non en scène. Nous avons demandé à certains de le photographier et de constituer ainsi un carnet visuel par pièces, de tout ce qui ressemble, dans leur logement, à un coquillage qu'il soit authentique, copie ou image. En somme, c'est en procédant - à ce que nous nommons - une autoethnographie élargie que nous avons saisi le sens contemporain des territoires ordinaires des coquillages, inscrivant notre réflexion dans une ethnologie de la culture matérielle.

\section{Faire entrer la nature chez soi}

Rien d'étonnant à voir dans les logements de citadins se côtoyer espèces végétales, animales et minérales, comme si l'habitat, symbole de la culture domestique, ne pouvait se suffire à lui-même, comme si aussi la nature devait se déployer autant en extérieur qu'en intérieur pour rétablir un équilibre. Peu importe son authenticité, cette nature 
est une nature revue et corrigée, adaptée, domestiquée, un semblant de nature, une copie, une image. Les coquillages n'échappent pas à cette logique qui interroge les frontières entre le naturel et le culturel.

S'ils incarnent dame nature, ne commencent-ils pas à être dénaturés, à perdre un peu de leur essence animale, dès lors qu'ils sont extraits de leur environnement, dès lors qu'ils franchissent le seuil d'un logement. Leur espace naturel n'appartient pas aux espaces intérieurs. La main de l'homme change leur aspect et par là leur statut. Récupérés, lavés et plus encore, polis, ornés, agencés, détournés etc., ils deviennent des objets. Leurs volumes, leurs spires, leurs nacres sont autant de qualités qui séduisent et expliquent leur mutation en bibelot. Les coquillages se transforment en ornement (parure, décor), quand bien même leur esthétique propre en fait des éléments décoratifs en soi. Ils cumulent également des fonctions utilitaires (cendrier, boîte, porte-savon, etc.), trouvant naturellement une place dans les intérieurs domestiques.

\section{Un bibelot tendance?}

Cette présence n'a rien de nouveau, et dans les hauts lieux de la société, depuis des siècles, elle s'accommode des tendances de la mode. Les coquillages ont intégré une économie de marché, sont rentrés dans le cycle des échanges: ils se vendent, ils s'achètent et ils s'offrent. Et comme tout article, leurs qualités sont vantées dans la presse ou les éditions spécialisées, des conseils sont prodigués. Ainsi on peut lire dans l'Encyclopédie de la décoration datée de 1964 que « Les collections subissent des modes. La Renaissance goûtait fort les cabinets de minéralogie et les coquillages montés en orfèvrerie. Sous la Restauration, on raffola des nacres montées en tabatières. Sous le Second Empire, des faux camées et des coquilles décorées. Aujourd'hui, on aime le coquillage pur, décapé et poli. » (1964 : 187). Ce rappel historique consacré aux collections prépare le lecteur à la recherche d'un style décoratif, aux suggestions qui suivront et qui insistent sur le respect de l'« harmonie des ensembles » (revêtement, mobilier, bibelots). Parmi les styles proposés, deux d'entre eux évoquent l'usage des coquillages comme « accessoire du décor » : le style Louis XV dont « la cheminée en marbre ou en bois [est] orné de coquilles » (1964 : 218) ou encore le style Napoléon III dont on trouve parmi les bibelots, « les nacres. Très courantes et très à la mode. Elles sont moulées sur cuivre doré; la coquille conserve sa forme naturelle. On trouve des encriers, des caves à odeurs, des vide-poches, beaucoup de coquillages décorés et de boîtes de carton bouilli, incrustées de nacre. » (1964: 230). Si nous ne pouvons mesurer l'impact de cette encyclopédie « populaire » sur les pratiques, en revanche, nous savons que les collections Marabout, « livre pratique », cherchaient à séduire un lectorat féminin, promouvant l'image d'une femme moderne sachant consommer à bon escient tout en étant à la page, soucieuse d'enrichir ses connaissances pour le bien-être familial. C'est donc à une classe moyenne émergente que s'adressent ces conseils de décoration, dont la légitimité trouve plutôt sa source dans l'histoire des mœurs de la haute société et moins dans celle du peuple. Les cendriers en coquille Saint-Jacques, les poupées régionales, les bibelots de bords de mer de tout genre qui se développent à la fin du xIx ${ }^{e}$ siècle (voir Charpy dans ce numéro) avec le tourisme balnéaire et relèvent d'une esthétique que l'on pourrait qualifier de kitsch, prêtant souvent à sourire (Souvenirs de

\section{Une coquille sur terre}

Ce coquillage est posé à même la terre, œuvrant à la combinaison d'éléments naturels. Discret mais bien présent, il s'impose comme un objet de décor parmi d'autres. Pourtant, ce cadeau marque aussi le lien avec la personne qui l'a offert. 


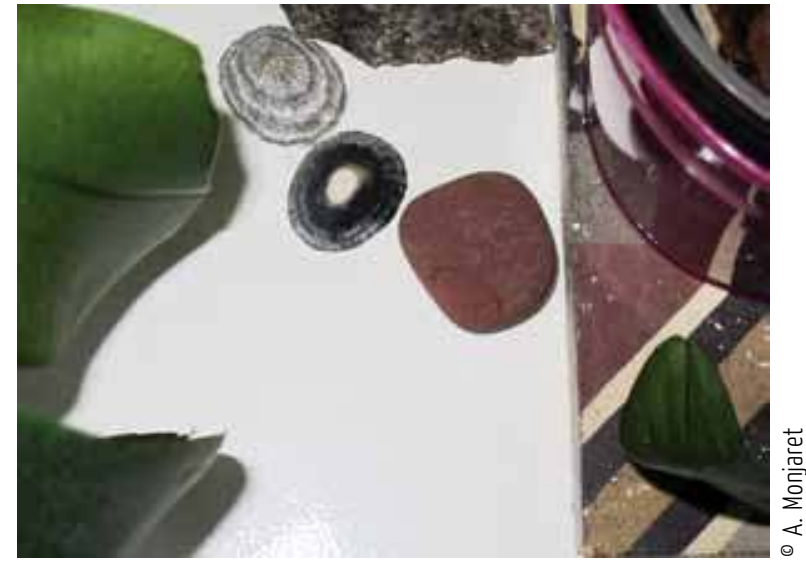

L'éloge à la nature

Ici les coquillages et le galet semblent avoir été négligemment déposés, mais de leur rencontre se dégage un équilibre qui participe de l'ambiance décorative de la pièce. rivages, 2001), semblent s'effacer de cette narration qui préfère valoriser le travail de l'orfèvre ou la beauté pure du corail ou du coquillage. L'éducation du consommateur ne semble pas ici renvoyée aux pratiques les plus communes.

Aujourd'hui, certains magazines ou boutiques de décoration réinventent les cabinets de curiosités - compositions sous cloche, jolis bric à brac baroques - clin d'œil aux grands explorateurs qui ont parcouru la planète, pour une clientèle aisée. Les coquillages sont ainsi mis en scène avec des vanités, des coraux, des animaux empaillés et autres articles évocateurs des voyages. Mais cette théatralisation ne réfère en rien aux saveurs ordinaires des vacances au bord de l'eau, aux plaisirs enfantins de rapporter chez soi son modeste butin estival banales coquilles, os de sèches, morceaux de verre polis par la mer, bois flottés ramassés sur la plage. C'est pourtant ce registre que nous avons le plus retrouvé dans les foyers visités.

\section{Nouveaux rivages pour coquillages}

Toutes les pièces semblent un refuge à coquillages: chambre d'enfant ou d'adulte, chambre d'amis, salon-salle à manger, cuisine, salle de bain, bureau, entrée et balcon. Mais toutes ne sont pas investies de la même façon, comme si certaines d'entre elles apparaissaient plus propices à accueillir ces « spécimens».

Ainsi, dans l'une des salles de bain observée, des coquillages vrais et faux cohabitent avec d'autres espèces. Les plus gros et plus beaux d'entre eux courent autour de la baignoire tandis que les plus petits, autour du lavabo. À proximité de ces derniers, une coquille tient lieu de porte-savon, un collier, deux étoiles de mer et un corail ont été placés dans une corbeille. Sur la coiffeuse, une jolie petite boîte en porcelaine vient compléter la précieuse collection. Vient s'ajouter à ces authentiques coquilles une série de patères ventouses - coquilles saint-Jacques, poissons et étoiles de mer -. Ce décor évoque en autant de petits signes la mer et les vacances au bord de l'eau. La salle de bain serait la pièce qui se prête le mieux à cette évocation. Lassociation de la salle de bain aux symboles balnéaires est somme toute assez commune, il suffit de feuilleter quelques magazines de décoration pour s'en convaincre. Ce qui l'est moins en revanche, c'est de trouver une profusion similaire de coquillages sur un balcon. Toujours chez la même personne, végétaux, minéraux et coquillages créent l'ambiance de son jardin suspendu. Cette fois cependant la mise en scène est différente, les coquillages sont mis en pot, comme le sont aussi les galets et plus évidemment les plantes vertes.

Ailleurs, c'est le séjour (salon et/ou salle à manger) qui est le lieu d'exposition privilégié des coquillages ou des fossiles. Ils se retrouvent dans le pot d'une plante verte, servant parfois de contrepoids. Mais ce sont plutôt les étagères de bibliothèque, la surface d'un meuble qui leur servent de présentoir. Bibelots isolés, coquilles posées ici et là ou regroupés sur une même surface pour parfaire une muséographie domestique, ou encore dessins qui les représentent fixés au mur, chaque intérieur a sa proposition décorative.

Dans les chambres à coucher, ils ne sont pas absents, mais ils se font davantage discrets, respectant l'intimité conférée à cette pièce de la maison. Cette discrétion tient à leur 
petit nombre comme à leur dispersion; elle tient aussi à leur préciosité (comme celle du collier de perles fines, qui plus est héritage familial, (voir Ciambelli dans ce numéro)) qui invite à les conserver pour soi et soi seul, à les réserver à l'intimité. La cuisine n'est pas oubliée. Ainsi, un collier fait de coquilles plates se trouve suspendu à un réfrigérateur; de micro-coquillages avec d'autres miniatures remplissent les petites niches d'un ancien casier d'imprimeur, exposé de façon centrale au mur.

Chacun dispose à sa manière ses trouvailles, mais tous signifient leur goût pour ces petits objets extraits de la nature. En entrant dans les espaces domestiques, les coquillages ont perdu un peu de leur part d'animalité - ils sont devenus « coquilles vides », comme dit l'expression. Leur forme aurait en quelque sorte pris le dessus sur leur contenu. Mais c'est bien parce que cette forme a été vidée d'un de ses sens premiers qu'elle a pu en cristalliser d'autres, susciter de nouvelles rhétoriques et se voir attribuer de nouvelles fonctions sociales, symboliques, esthétiques...

\section{L'esthétique du seau ou de l'éprouvette}

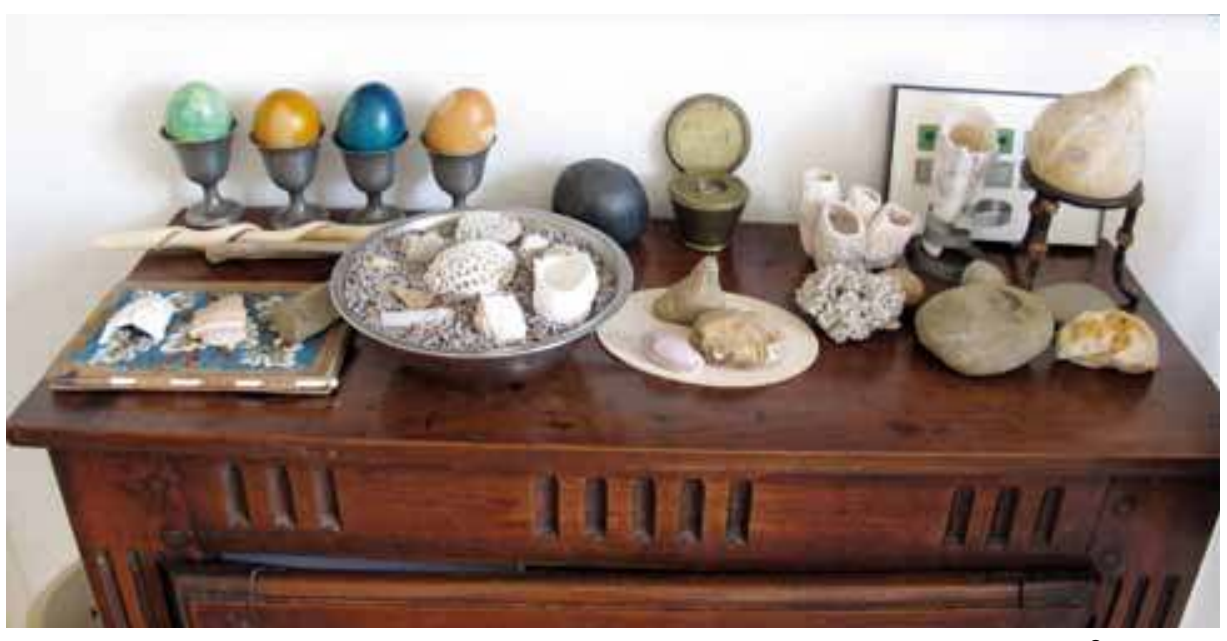

${ }^{\bullet}$ A. Monjaret

Venus de pays reculés, lointains ou proches, les coquillages et les fossiles peuvent être autant des trouvailles de promenade, extraites de leur environnement naturel (la mer et ses bords ou la terre) que des marchandises achetées ou reçues en cadeau. Sorties de leur contexte, elles deviennent des objets manipulés et ce, même si leur état brut, au naturel, est souvent préféré ici dans l'élaboration de petites mises en scène. Certaines fois, ce sont de faux coquillages qui trônent sur des étagères. Il arrive qu'ils donnent l'impression d'y avoir été posés négligemment, comme abandonnés. Tous cependant participent au décor des intérieurs domestiques. Cultivés et esthétisés, ils deviennent l'expression d'un goût du beau. Quelle forme cette esthétisation prend-elle? Quelle façon avons-nous de les thêâtraliser? C'est ce à quoi nous nous intéresserons ici.
Cabinet de curiosités Ce regroupement d'objets apparemment disparates - série d'œufs de Pâque, coquillages, bois, poids, coloquintes, carnets perlés, etc. -, forme un ensemble esthétiquement harmonieux. II évoque une sorte de cabinet de curiosités qui met en scène des cadeaux de famille, d'amis, de voisins ou encore des souvenirs rapportés de voyages. II apparaît comme un concentré d'histoires de vie, offert au regard. 


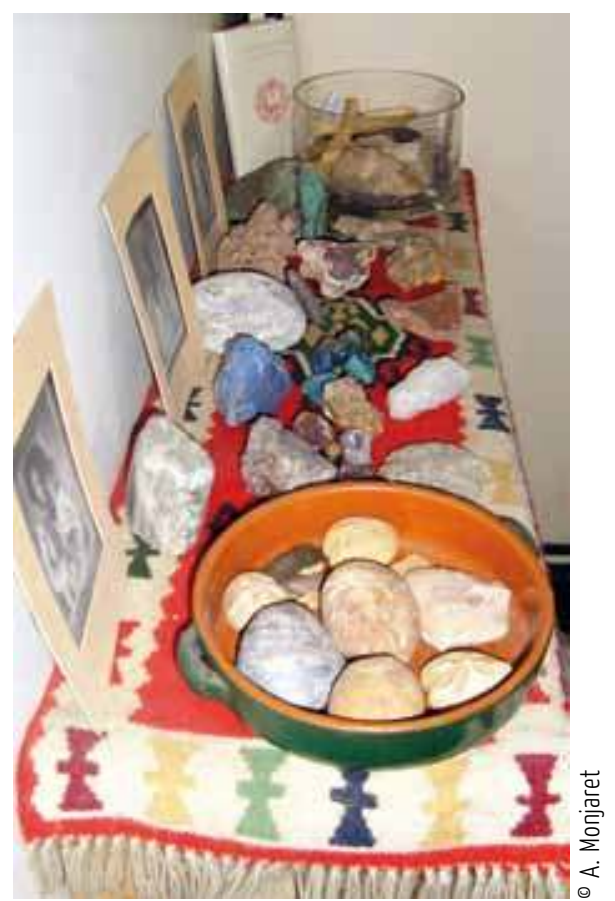

Une association minérale

Sur cette étagère réservée au monde minéral, la présentation des fossiles et des pierres oscille entre le modèle de la collection ou la simple recherche esthétique. Elle montre les manières de réunir des semblables et surtout dévoile des logiques profanes de classement.

\section{Mises en scènes pour coquillages}

Les coquillages ou fossiles sont souvent exposés en vrac, amassés, de façon indifférenciée, dans une corbeille, un pot de terre ou dans toutes sortes de récipients qui ressemblent de près ou de loin à un seau en plastique, celui-là même qui sert aux ramassages des coquillages. C'est ce qui nous a incités à qualifier ce mode de présentation d'esthétique du seau, il nous semble que l'on y retrouve des principes similaires dus à un effet de masse. Cet amoncellement forme un joli petit bazar qui n'est cependant pas au goût de tout le monde, certains préfèrent des installations plus ordonnées, ainsi ces oursins fossilisés, trouvés dans les champs avoisinants, qui ont soigneusement été placés en cercle dans un plat en terre. Cette présentation valorise l'unicité de chacun des fossiles, tout en incitant à leur comparaison. D'autres effets sont recherchés pour mettre en valeur les trésors réunis. Celui de transparence l'est particulièrement. Cloches ou anciens encriers en verre, aquariums boules, tubes à essai ou autres flacons, boîte en plastique, cube en plexiglas sont des récipients idéaux. Ils permettent toutes sortes de compositions, de l'entassement pensé à la reconstitution miniature d'un bord de mer. Ces mises en scène font référence à des registres a priori propres aux coquillages, dès lors qu'ils ont franchi le seuil d'un logement.

\section{La sacralité du savant}

La mise sous cloche ou en vitrine rappelle le reliquaire; en sacralisant l'objet, elle en souligne la préciosité, ici sa valeur est le plus souvent affective. Mais, ce procédé décoratif évoque également les cabinets de curiosités, quand il n'apparaît pas comme une référence au registre du scientifique. Le tube qui contient un mélange hétéroclite de plumes, pierres et coquillages ou encore des coquillages sur leur sable (Zisman, 2004b), la boîte, écrin cotonneux dans laquelle sont disposés des micro-fossiles rappellent le dispositif de prélèvement du scientifique, l'échantillon qu'il ramène d'une expédition (voir Dumoulin dans ce numéro). Il s'agit autant de préserver que de voir pourtant ici ce n'est pas le travail qui l'emporte, c'est le plaisir d'admirer. L'éventuel regroupement par famille n'est pas non plus sans rappeler les classements typologiques des espèces, effectués par les chercheurs ou les collectionneurs, amateurs passionnés. Les vitrines appartiennent au mobilier d'exposition des collections mais l'usage que nous en avons observé s'apparentait plus à "l'avoir de l'enfance » (Vincent, 2005 : 185). Les planches dessinées de malacologie rentrent dans la pratique du scientifique mais en pénétrant dans les intérieurs domestiques, les plus anciennes s'affichent comme des dessins d'art, partageant l'espace d'exposition avec des peintures plus contemporaines. Il arrive que ces décorations soient de discrets indices d'un attachement à un métier. Mais le plus souvent, l'évocation scientifique, n'est que le fait d'un jeu de représentations, conscient voire inconscient, qui conduit à une forme de sacralisation du travail du savant. Ce monde professionnel, lointain pour le quidam, imprègne subrepticement notre quotidien, rend esthétiquement familier un objet de science. Il reste que la plupart du temps, nous ne maîtrisons 
aucunement la terminologie qualifiant les espèces que nous possédons et préférons l'emploi générique de « coquillage » pour les nommer ou l'emploi d'une terminologie commune qui désigne les coquillages que nous consommons: bigorneaux, coques, escargots de mer, oursins, etc.

C'est l'objet-coquillage qui intéresse plus que sa référence malacologique. En nombre, ils deviennent également matière à création, servent à la composition de tableaux originaux. Ces vrais coquillages côtoient aussi de faux coquillages sans que cela n'apparaisse gênant. Bibelots en céramique, en plastique ou autres matières, bordures de cadre en plâtre moulé imitant la plage, les coquillages sont dessinés, copiés, réinterprétés. Ils sont sources d'inspiration. Des fibres crochetées prennent la forme de drôles de coquilles à moins que ce ne soit de fossiles. Ainsi, le coquillage se transforme en objet - objet de curiosité sinon de science, objet-souvenir ou bel objet. L'art du coquillage est aussi un art décoratif.

\section{Voyage intérieur}

Par-delà cette fonction esthétique, les coquillages et fossiles renvoient, d'une façon récurrente, à la mer et au voyage. Ils en sont les symboles. Pourquoi une si forte imprégnation de ces représentations est-elle possible? Comment expliquer cette incarnation? Est-ce parce que les coquillages circulent aisément, sont facilement transportables? Dans tous les cas, ils invitent celui qui en possède à un voyage intérieur.

\section{L’appel du large}

Avec les coquillages, c'est «le monde qui vient à soi ». Ils incarnent les souvenirs de voyages que l'on aime à se remémorer mais aussi des «souvenirs de voyages - que l'on n’a pas faits » (Chevalier, 2001 : 510). Contrairement à d'autres bibelots, leur seule évocation les sort de l'ordinaire en stimulant un imaginaire: on se prête à rêver de vacances au bout du monde - d'exotisme - ou plus modestement de vacances à la plage - et de familier -, une évasion en quelque sorte sans voyage effectif, un appel du large sur place, à portée de mains ou d'oreilles.

En portant une conque à l'oreille, ne pouvons-nous entendre le bruit de la houle et être transporté loin de chez soi, sentir l'espace d'un instant le plaisir des bords de mer vécu ou rêvé? Ici c'est l'esprit qui est en voyage. Il suffit d'un seul spécimen pour provoquer l'agréable sensation d'être ailleurs. Ce processus se passe de la rhétorique d'un ancrage géographique ou temporel tangible, la provenance des coquillages et la circonstance de leur acquisition semblent n'avoir alors que peu d'importance.

\section{Les vacances en tube}

Ce principe de l'éprouvette contribue à la visualisation d'un concentré miniature de trésors de voyage. Un seul coup d'œil suffit à la réminiscence des vacances et de leurs bons moments. Mais il arrive que progressivement le souvenir précis de la destination de ces coquilles et de ces fruits d'eucalyptus s'efface, laissant place à l'unique fonction décorative. 


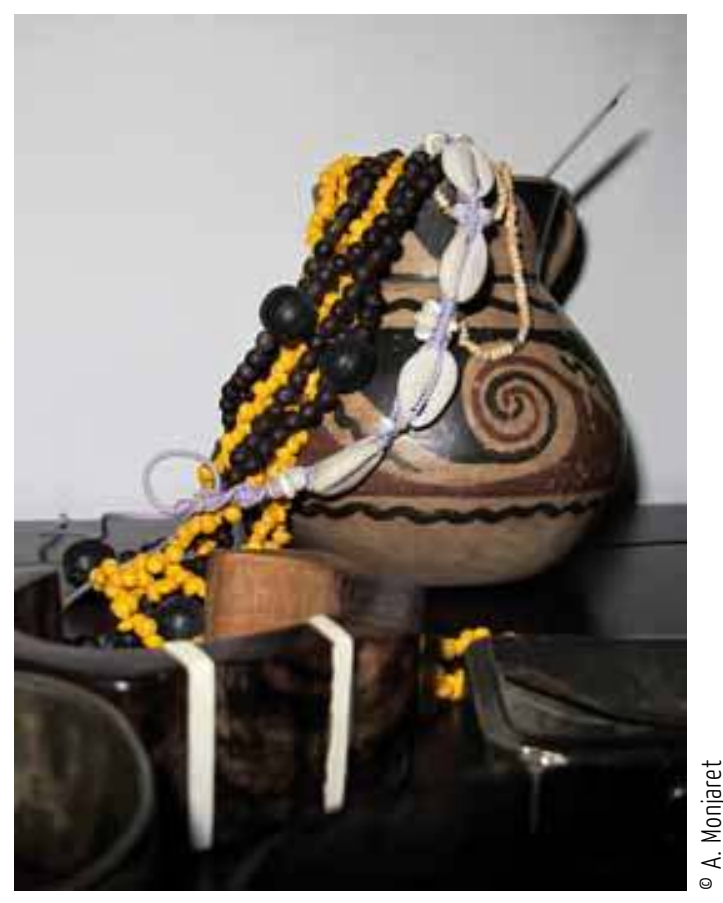

Le pot à bijoux

$\|$ y a des pots qui deviennent des présentoirs à bijoux. Mais ici les colliers et le bracelet, offerts par des amis, ont été installés plus par souci esthétique que pratique.

\section{L'autel des souvenirs}

Pourtant, si on s'attache plus à fond au sens et à l'essence des coquillages chez soi, on s'aperçoit vite qu'ils ont un autre rôle, celui de permettre de se remémorer des gens, des lieux et des moments. Ils sont les traces matérielles et mnémoniques d'une balade, d'un voyage, d'une mission, des traces qui constituent une topographie mémorielle qui ne demande qu'à être cultivée, au risque de s'effacer des souvenirs personnels et de n'être plus qu'un cliché du voyage.

Cadeaux d'amis ou de famille, coquillages rapportés d'un voyage, ils renvoient à la mémoire de l'autre et de soi. La façon dont ils prennent place dans l'espace, ostensiblement ou discrètement, souligne les formes de la relation que nous entretenons avec eux. En effet, impossible de faire abstraction de l'histoire qu'ils portent, la leur et celle de leur propriétaire. Certaines de ces mises en scène domestiques ne sont d'ailleurs pas sans rappeler celles de l'autel ou de la boîte à trésors.

\section{Le goût de l'enfance}

La boîte à trésors apparaît comme une forme de reviviscence de l'enfance (Muxel, 1996). Elle concentre un bric à brac dans lequel se cachent des secrets et se conservent des bribes d'histoires de la vie ordinaire ou estivale. Ces petites collections d'adulte ont gardé leur âme d'enfant. Si elles renvoient à des souvenirs précis et personnels, lointains et plus récents, elles incarnent certaines saveurs des vacances, et parmi les trésors amassés, les coquillages rappellent plus évidemment les vacances passées à la mer; leur petite taille permet de ramener sans trop s'encombrer un peu de vacances dans nos bagages. Des souvenirs plein la tête, ils contribuent à ce que le voyage se poursuive, jusqu'à n'être plus, le temps passant, que l'archétype de menus bonheurs intemporels, une invitation à la rêverie où se mêlent images de soleil et de plage et images de l'enfance, tout un monde ainsi réactivée par les saveurs sucrées d'un « Roudoudou».

\section{Leçon de choses, pour que les rêves de coquillages ne meurent jamais!}

C'est bien parce que les coquillages sont porteurs de ces multiples valeurs que, de génération en génération, la transmission des usages qui leur sont associés semble devoir s'imposer. Cette transmission repose sur l'envie de faire vivre à ses enfants des moments marqués par des faisceaux d'émotion et de les revivre à travers eux. La mémoire familiale est ainsi cultivée. C'est un héritage matériel et immatériel qui se perpétue. 


\section{Avoir de « bonnes lectures »: instruction, clichés et sensations}

La littérature enfantine, outil pédagogique par excellence, apparaît comme une bonne entrée en matière pour s'approprier quelques clichés de vacances et surtout se familiariser avec quelques notions élémentaires de sciences naturelles. Prenons l'exemple de la collection des « Caroline » qui s'adresse à un jeune lectorat. Parmi ces volumes richement illustrés, deux d'entre eux nous intéressent particulièrement: Caroline à la mer (Probst, 1989) relate des vacances en bord de mer quand Caroline et le mystère du chat fossile (Probst, 1994) propose le récit d'un fossile.

Le premier nous plonge dans des souvenirs d'enfance racontant les vacances d'une joyeuse bande (chatons, chiots, ourson, jeune tigre et petite panthère) qui tient compagnie à Caroline dans sa « jolie villa » en location « tout au bord de la plage ». L'une des séquences reprend la classique scène du ramassage des coquillages, seau en main:

« Youpi (chien), lui, préfère se promener sur l'immense plage, droit devant lui, droit vers l'horizon. Que fait-il donc? Il ramasse des coquillages pour son amie Caroline. En voici un... un autre... et puis un autre encore... Tout à ses recherches, Youpi ne voit ni le panonceau qui indique l'heure des marées, ni l'eau qui est en train de monter, monter, monter... ».

Il se laisse surprendre.

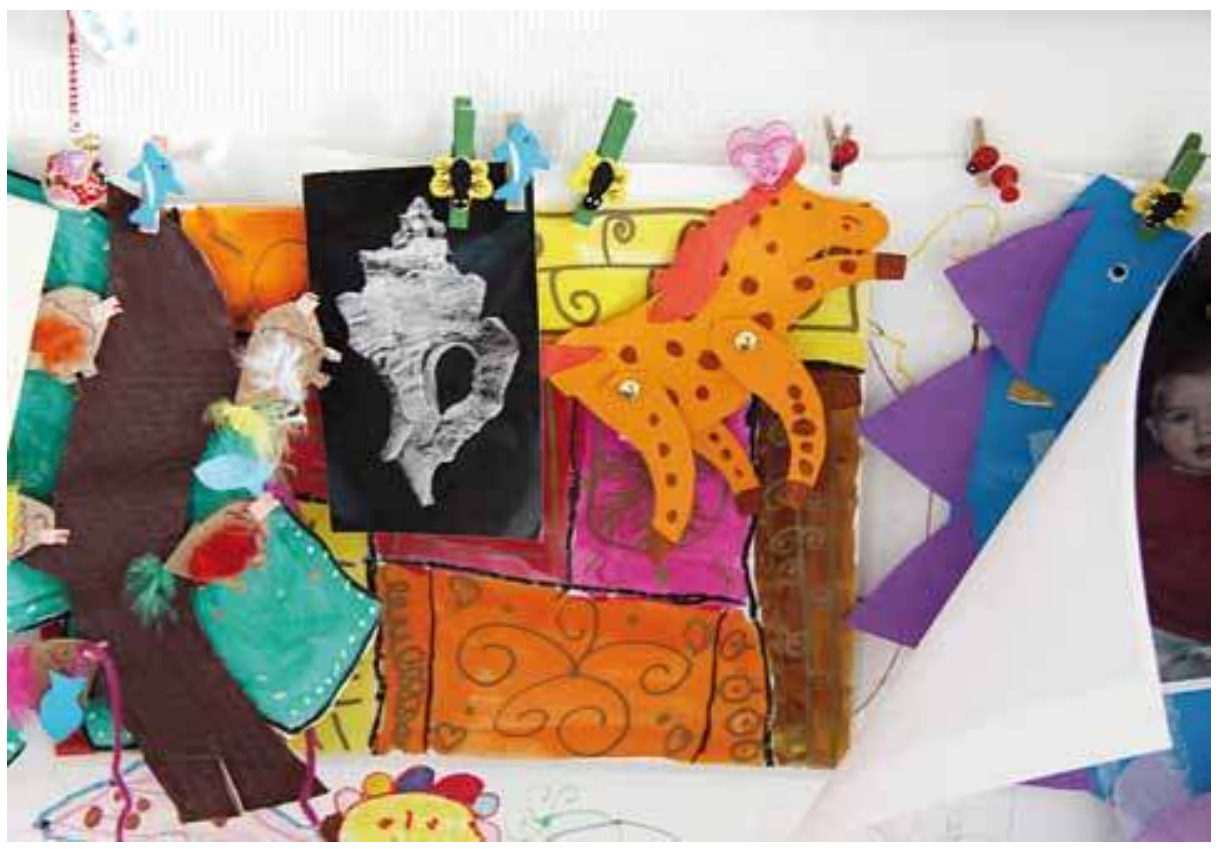

o Pierre Lozouet

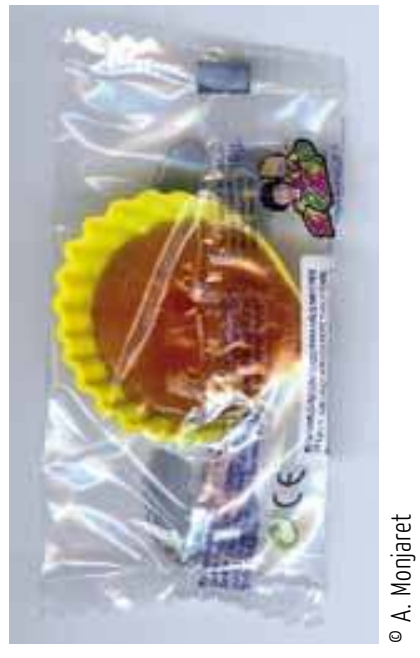

\section{Roudoudou, bon à manger}

Le Roudoudou, vendu aujourd'hui dans des coques plastiques, incarne doublement les saveurs de l'enfance, celles de la douceur des sucreries et des plaisirs de la mer. Cette rencontre réactive l'esprit suave des vacances. Le roudoudou appartenait aux recettes familiales: les coquilles (coques, amandes de mer, etc.) nettoyées étaient garnies d'un coulis coloré et aromatisé qui en durcissant devenait ce bonbon que les enfants léchaient avec gourmandise.

\section{Coquillage accroché à un fil}

Les coquillages, réels ou imagés, appartiennent aux univers enfantins, indifféremment, préservés pour soi dans des boîtes à trésors ou offerts à tous dans des vitrines. Parfois, une photographie suffit à marquer leur présence. Accrochée à un fil, à la manière d'un linge, l'image noir et blanc de ce coquillage dénote parmi les autres papiers découpés et dessins, tout en semblant se perdre dans la masse colorée des souvenirs. 


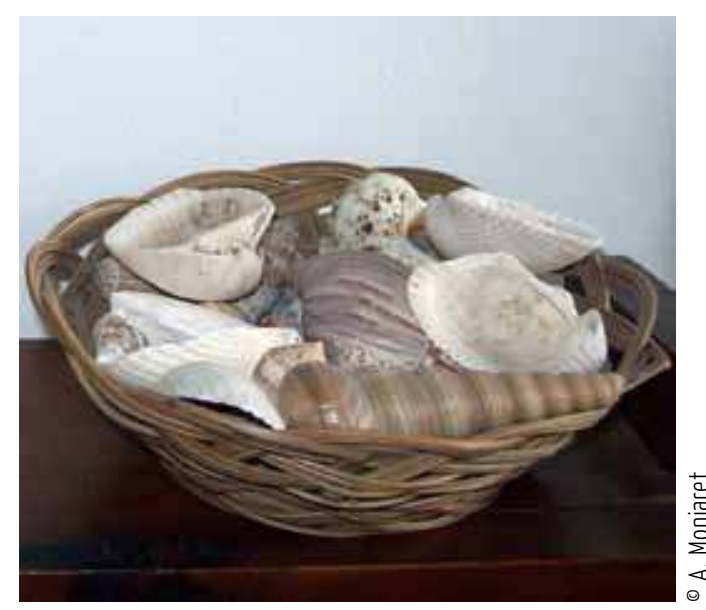

Histoires de bord, de cadre et de mer

Les corbeilles d'osier, comme

les pots de terre, les bols, etc. aux formes assez similaires, sont de ces contenants communs qui accueillent toutes ces coquilles ramenées des balades en bord de mer, samoncelant progressivement après chaque retour.

De fabrication industrielle, ce cadre possède pourtant la naïveté des objets confectionnés de bric et de broc par les enfants. Il est la démonstration que les coquillages servent souvent à convoquer des imaginaires associés à l'enfance et en particulier ici celui qui renvoie aux vacances à la mer.

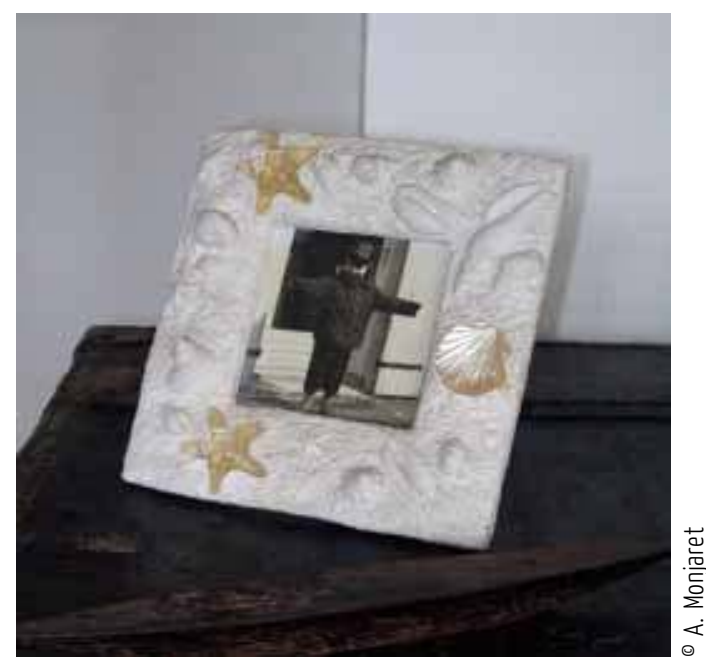

La dernière séquence revient sur le départ, illustré par le chargement dans la voiture des cartons qui débordent de coquillages:

« Aujourd'hui, Caroline et ses amis rentrent chez eux. Les vacances sont finies. (...) "L'été prochain nous reviendrons, promet Caroline. En attendant ce moment, nous écouterons les histoires merveilleuses que la mer nous racontera dans les jolis coquillages que nous avons trouvés..." ».

La leçon est claire.

Le second titre raconte la mystérieuse découverte d'un fossile à travers les péripéties de la petite bande d'amis de Caroline. C'est dans une carrière que se noue le cœur de l'intrigue puis au muséum d'histoire naturelle que se poursuit l'aventure. Nous ne rentrerons pas dans le détail du récit, ce qui nous importe ici est de montrer que l'usage de clichés concernant le monde des savants sert de support à une narration pédagogique. Les extraits ci-dessous permettent d'en mesurer la teneur: « "Dépêche-toi, Pouf, viens voir ce drôle de caillou! Pouf prend son élan, dérape et [...] "Si vous ne m’aviez pas appelé, je n'aurais pas couru, et si je n’avais pas couru, je n’aurais pas dérapé. Tout ça pour voir un caillou! — Justement, Pouf, rectifie Youpi, ce n'est pas un caillou, c'est un coquillage! —Un coquillage? Tu te fiches de moi, Youpi, nous ne sommes pas au bord de la mer! (...) "C'est un caillou tout en étant un coquillage, déclare Caroline, ou, si vous préférez, un coquillage devenu un caillou au cours des temps!

— La mer venait donc jusqu'ici?

— Oui, mais il y a longtemps, très longtemps, des millions d'années. Ce coquillage en est la preuve. (...)

- Allons faire une visite au Muséum, nous y verrons beaucoup d'objets qui racontent, mieux que moi, l'histoire de notre Terre" propose Caroline. »

Les enfants apprennent ainsi à distinguer le coquillage de la pierre.

\section{Les jolies colonies de vacances}

Cet apprentissage peut se poursuivre en classe de mer, en colonie ou en famille, autant de moments propices à la découverte des « Coquillages et crustacés », des « Rochers, coquillages et crustacés », aux plaisirs de «Plage, coquillages et enfantillages ». Ces expressions qui désignent les colonies de vacances au bord de l'eau sont révélatrices des activités qui s'y pratiquent.

Les enfants s'initient aux joies de la nature en compagnie d'adultes qui se replongent dans leur propre enfance, apprennent le respect de l'environnement, savourent le silence de la contemplation, forment leur regard et leur capacité de concentration quand ils partent ramasser des coquillages et coquilles, et surtout prennent conscience de la distinction entre le vivant et le mort. Laver, tremper, sécher, trier, peut-être classer, parfois cuire, ils s'occupent de leur récolte pour la ramener ensuite chez eux.

De retour à la maison, ils peuvent faire partager ce trophée avec leur camarade de classe ou avec des membres de leur famille. 
Marie Rouanet se souvient de ces amies qui avaient quitté la ville pour l'été et « qui revenaient (...), avaient des souvenirs de colonie, sortaient de leur boîte des graines inconnues, des coquillages, de nouvelles images. » (Rouanet, 1990 : 283). Parfois le trophée se fait plus imposant au point que sa masse devienne embarrassante, et ce d'autant plus qu'elle ne trouve pas à proprement d'intérêt aux yeux de ceux qui n'ont pas vécu ces vacances. Seuls les plus beaux ou les plus petits seront conservés, venant compléter la collection déjà constituée. Rangés dans une boîte, posés sur une étagère, les coquillages appartiennent désormais à l'univers de l'enfant, et occupent ce microcosme qu'est sa chambre.

\section{Affaires de famille...}

En intégrant l'espace familial, ils acquièrent un statut, qui se rattache à l'histoire de famille. Que représentent-ils pour ses membres? Ne deviennent-ils pas des repères identitaires qui redessinent les parcours de vie, en marquent les étapes? Que nous révèlent exactement ces « objets d'affection » (Dassié, 2012)?

\section{Le monde en miniature des enfants}

Dans leur chambre, à la manière de leur boîte à trésors, les enfants « inventent des décors qui mobilisent aussi bien leur curiosité que leurs souvenirs et leur imagination, ainsi que leurs multiples désirs d'identifications familiales et sociales. Ils réalisent des compositions qui tiennent en partie du fragment autobiographique, de l'autoportrait, de l'archive familiale, du trésor pris sur le monde des objets de consommation et celui des choses de la nature. Les pierres et les coquillages, souvent accompagnés de fossiles, racines, plumes d'oiseaux ou débris d'os, figurent dans toutes les collections d'enfants en tant qu'échantillons de l'univers sous ses aspects terrestre et aquatique et comme témoins de ses origines. » (Vincent, 2005 : 187). Ainsi, la chambre renferme des « entassements de fragments prélevés sur le monde » (Vincent, 2005: 187).

Les enfants n'ont que faire des principes de la collection, la leur en serait même l'antithèse. S'ils rassemblent bien les objets, ils apprécient surtout leurs amoncellements. Peu importe qu'ils aient une « idée directrice » qui en constituerait « le premier pas, le geste créateur », peu importe qu'ils en établissent des séries originales, ils préfèrent « une foule disparate » et ce, même si cette «prolifération » est déconseillée car elle est «le point optimal de l'esthétique » à ne pas dépasser au risque de détruire l'effet recherché (Encyclopédie de la décoration, 1964 :184-185). Peu importent également les modalités de leur exposition. La vitrine de coin est là plus pour entreposer ses trésors que pour véritablement les donner à voir. Elle fonctionne comme la boîte à trésors, elle cache plus qu'elle ne montre. Cette collection est à soi, pour soi, et non pour les autres. C'est sans doute ce qui explique le goût plus prononcé des enfants pour les miniatures, les petites

\section{Décor de balcon}

Sur ce balcon de banlieue parisienne, plantes, galets, coquilles - appel ou rappel de la nature dans un univers urbain - partagent un territoire commun tout en préservant leur propre espace. Les regroupements par catégorie se font rigoureux et ludiques à la fois. Les pots remplis de coquillages évoquent d'une certaine manière le seau de plage que les enfants aiment à garnir de leurs trouvailles. 


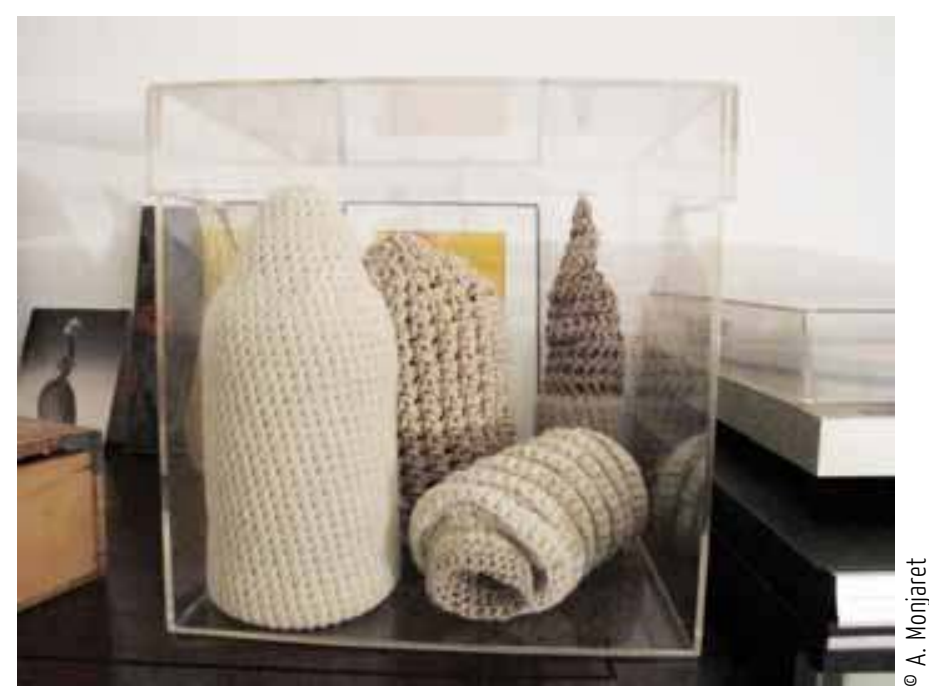

La nature à l'œuvre

La plasticienne, Delphine de Luppé, semble s'inspirer de la nature. Elle réinterprète les formes qu'elle y rencontre et propose des versions de coquillages ou fossiles crochetés dans des matières fibreuses brutes quélle met sous cloche. Cet enfermement dans une boîte de plexiglas renvoie à la fois au principe de mise en exposition qui préside à la création artistique et au principe de transparence qui commande le travail scientifique.

Les perles de ma grand-mère Dans les familles, il est coutume d'offrir à sa fille ou sa petite fille, un collier de perles, ses perles. Cet héritage doit être respecté pour que le cycle ne soit pas interrompu, que le fil générationnel ne soit pas rompu: les perles doivent être portées afin qu'elles ne meurent pas..

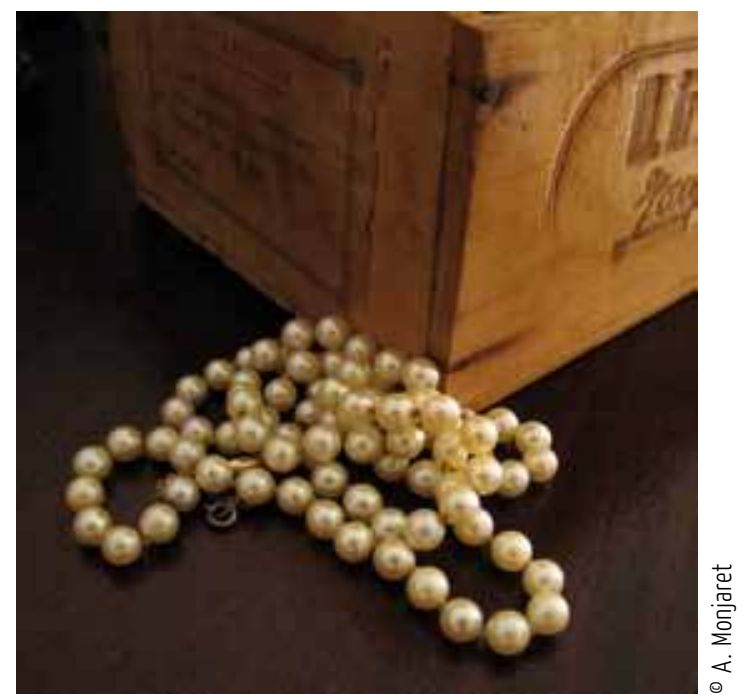

choses qui peuvent les suivre. Nous comprenons mieux alors pourquoi les coquillages les séduisent tant. Ils cristallisent une part de leur histoire, les mémoires individuelle et familiale, le souvenir d'un été passé avec un père, le cadeau offert par un proche de retour de voyage, etc.

\section{Des cadeaux, miroir de la famille}

Les coquillages sont bel et bien une affaire de famille. Qu'ils soient trouvés sur le sable d'une plage, achetés en boutique ou confectionnés à la main, dès lors qu'ils sont offerts, donnés en héritage, ils racontent l'histoire du lien familial.

De leurs vacances les enfants petits et grands aiment rapporter des cadeaux souvenirs à leurs parents (Chevalier, Monjaret, 2001). Ils ont ce goût pour les bibelots kitsch qu'ils abandonnent parfois au sortir de l'enfance. C'est ainsi que Marie Rouanet raconte comment ce qui avait fait son « bonheur devenait puéril »: "C'est de moi-même que je tus mon goût pour les coffrets de carton recouverts de coquillages nacrés où il y avait écrit "Souvenir de Valras", pour la boule transparente où il neigeait sur Notre-Dame de Lourdes. » (Rouanet, 1990 : 341).

Pourtant au-delà de leur esthétique, ils témoignent des déplacements des proches, annoncent « de manière transparente leur lieu de provenance », sont « reconnus de tous dans l'instant », (Halitim, 1996 : 236) et surtout ils œuvrent à leur partage avec ceux qui ne sont pas partis. Nadine Halitim qui a rencontré nombre de familles populaires dans la banlieue de Lyon insiste sur cette fonction des souvenirs de voyages. « Au-delà du caractère affectif du cadeau, le souvenir rapporté "d'ailleurs" constitue une invitation symbolique au voyage. Il permet de faire participer les parents au voyage de l'enfant. » (Halitim, 1996 : 236), un voyage que ces derniers n'ont pas forcément les moyens de se payer. Le cadeau apparaît comme une « plus-value culturelle » qui est ainsi transmise (Halitim, 1996 : 240).

C'est à travers ces cadeaux que les mémoires, individuelle et familiale, se construisent et se cimentent, que les identités des exilés se reconstruisent. Selon Nadine Halitim, «si le pays visité est celui de l'origine familiale, le souvenir rapporté par les enfants permet une réinscription dans la culture géographique spécifique de la famille ». L'auteure prend l'exemple de roses des sables et de coquillages rapportés d'Algérie - « ça vient de la mer de là-bas. » ou de petits coquillages qui viennent d'Espagne ou d'autres du Cameroun - «parce que ma mère vit au Cameroun pour le moment ». Il s'agit également de compositions faites à la main comme ce cadre décoré de coquillages, de sable, des grains de melon ou de pastèque, offert par son neveu à sa tante Kabyle (Halitim, 1996 : 240-242, 257-258). Ici, la morphologie commune de ces espèces n'aide pas à une reconnaissance spontanée de leur provenance, seul le récit de voyage peut y contribuer. Dans tous les cas, chacun de ces cadeaux va être habité autant par son 
donateur qui y projette une « représentation culturelle » (Halitim, 1996 : 240) que par son donataire qui se l'approprie et réinvente un ancrage avec son pays d'origine.

Pour ceux qui ne sont pas concernés par l'exil, ces souvenirs de voyage jouent un autre rôle, celui d'intégrer à domicile une image de l'altérité quand ce n'est pas d'exotisme. Dans tous les cas, ils deviennent des « ego-objets » (Zisman, 2004a), pouvant alimenter un « egomusée » (Muxel, 1996 : 164) qui ne trouve sens que dans la narration qui les accompagne.

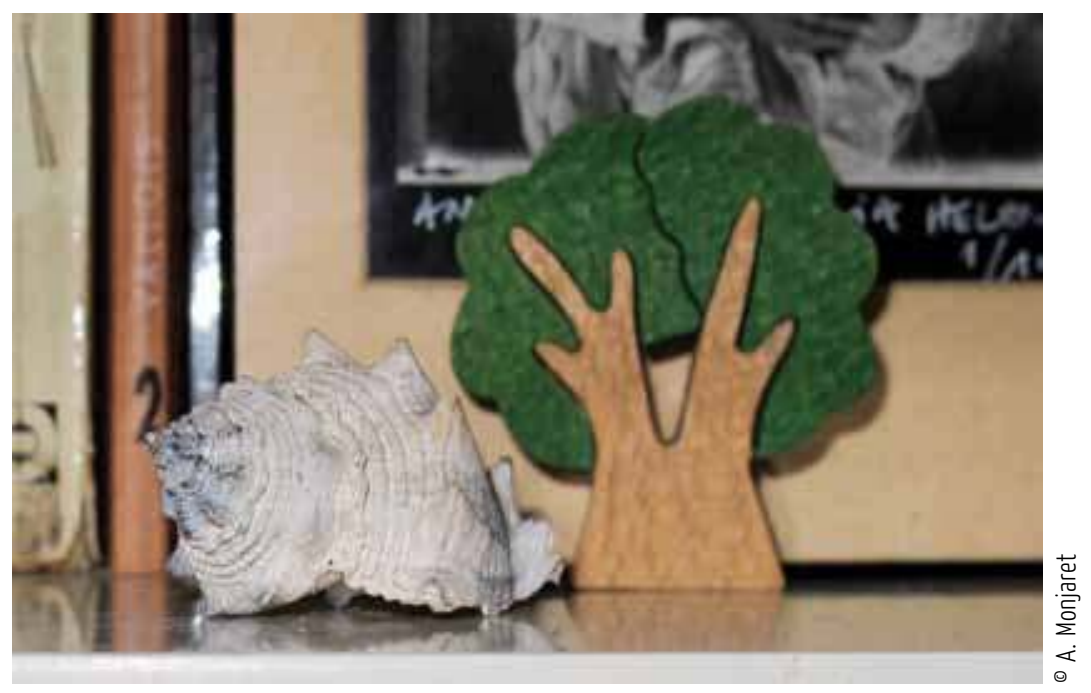

\section{L’arbre et le coquillage}

Les rencontres sont parfois étranges: sur une étagère de bibliothèque, un arbre de bois côtoie un coquillage, se jouant de l'échelle de grandeur. Cette mise en scène plus que d'autres a pris l'aspect de celle d'un autel.

\section{L'âme des coquillages en voyage}

Les coquillages, souvent si communs, parfois insolites, se fondent donc dans le décor domestique. Ils s'insinuent subrepticement dans nos vies, s'amoncellent, de ci de là au fil du temps, dans toutes les pièces de la maison, occupant plus ou moins d'espace. Leur petite taille n'y change rien. Il semble difficile de s'en débarrasser. Il en reste toujours, un ou deux, dans un coin, ce qui leur confère une certaine pérennité, un brin d'éternité, au moins symboliquement.

Les coquillages auraient-ils une âme? Une âme voyageuse? Celle-là même qui aide à partir ailleurs. Ils nourrissent un imaginaire des vacances, du voyage. Mais ce voyage a la particularité de se dérouler sur place, d'avoir lieu dans son environnement ordinaire. Ainsi, il allie le familier et l'exotique, le proche et le lointain, le commun et le scientifique. En stimulant un voyage intérieur, un voyage en intérieur, les coquillages se vivent, se ressentent plus qu'ils ne se disent ou ne se racontent.

(Trans)portent-ils l'âme de ceux qui les ont recueillis? Ce qui expliquerait en partie l'attachement qu'ils engendrent. Leur présence apparaît suffisamment nécessaire pour que s'opère, d'une génération à l'autre, la transmission des valeurs et des pratiques qui leur sont associées. Ils deviennent symboles des migrations individuelles et/ou familiales, l'un des ports d'ancrage de l'enfance auquel on se rattache. Les coquillages ou plus exactement les coquilles portent la mémoire de lieux, de moments et de gens. Ils habitent nos intérieurs et nous les habitons en retour. 


\section{NOTES}

Photo d'ouverture: Esprit de la collection. Le casier d'imprimeur est idéal pour présenter de petits objets; il peut prendre place dans différentes pièces du logement: il trône ici dans une cuisine. Les coquillages de petites dimensions permettent de se prêter avec délice aux plaisirs enfantins de la miniature et de la collection. (Cliché P. Lozouet)

1. Je les remercie tous chaleureusement d'avoir contribué à cette enquête.

\section{RÉFÉRENCES}

Bromberger, Ch. (dir.) 1998 Passions ordinaires. Football, jardinage, généalogie, concours de dictée. Paris: Bayard éditions/Hachette.

Chevalier, S. 2001 (1998) Destins de cadeaux. In S. Chevalier \& A. Monjaret (dir.) Les cadeaux: à quel prix? Ethnologie française (4) Paris: PUF, 506-514.

Collectif, 2001 Souvenirs de rivages. Bibelots et images du tourisme balnéaire. Catalogue d'exposition des Musée portuaire de Dunkerque, Musée portuaire de Dunkerque, Château-Musée de Dieppe, Musée de l'Abbaye Sainte-Croix (Les Sables d'Olonne), Musée national de la Marine (Toulon), Musée archéologique d'Istres, Musée Louis-Senlecq (L'Isle L'Adam). Paris : Édition Somogy.

Dassié, V. 2010 Objets d'affection, une ethnologie de l'intime. Paris: Éditions du CTHS.

Deniot, J. 1986 Le décor textile. Les murs et la table en milieu ouvrier. Ethnologie française (3) Paris: PUF, 319-334.

Encyclopédie de la décoration 1964 Choisir les matériaux, décorer, transformer, parachever. Tome II, Verviers (Belgique): Des presses de Gérard \& Co (Collection Bibliothèque Marabout).

Halitim, N. 1996 La vie des objets. Décor domestique et vie quotidienne dans des familles populaires d'un quartier de Lyon, la Duchère. 1986-1993. Paris: L'Harmattan.

Gérôme, N. 1981 Les maisons de pêcheurs de Saintonge. Ethnologie française (1) : 37-46.

Muxel, A. 1996 Individu et mémoire familiale. Paris : Nathan.

Rouanet, M. 1990 Nous les filles. Paris: Éditions Payot.

Segalen, M. 1987 Objets domestiques de la vie ouvrière. Transmissions et ruptures dans les familles de Nanterre 1920-1960. Ethnologie Française (1): 29-37.

Vincent, O. 2006 Entre enfance et adolescence: l'âge des petites collections. In Noël Barbe et Emmanuel Jallon (dir.) Vous avez dit: «Âges de la vie? Pluriel Texte (coll. Collection des musées départementaux de la Haute-Saône): 180-198.

- 2011 Collectionner? Territoires, objets, destins. Paris: Créaphis éditions.

Zisman, A. 2004b Ramener le désert à la maison. Quand le sable devient objet. In Véronique NahoumGrappe et Odile Vincent Le goût des belles choses. Ethnologie de la relation esthétique, Paris : Éditions de la Maison des sciences de l'homme (Cahiers d'Ethnologie de la France 19): 165-173.

— 2004a Légo-objet. Ethnologie française (1) : 139-146.

\section{Littérature enfantine}

Probst, P. 1994, Caroline et le mystère du chat fossile. Paris: Hachette jeunesse.

- 1989 Caroline à la mer. Paris: Hachette Jeunesse. 


\section{RÉSUMÉ}

Espaces d'espèces. Les coquillages ordinaires chez soi ou une invitation à un voyage intérieur. Cet article s'intéresse à toutes les sortes de coquilles (ou plus communément dits « coquillage ») qui occupent nos univers domestiques. Il interroge entre autres le sens des usages qui leur sont associés. Les coquillages conjuguent à la fois une fonction esthétique et une fonction mnémonique. Ils ont la particularité de s'insinuer subrepticement dans nos vies. Ils s'accumulent au fil du temps de ci de là dans les pièces de nos logements, au point qu'il semble parfois difficile de s'en débarrasser, ce qui leur confère un brin d'éternité. Ils habitent nos intérieurs tout comme nous les habitons en retour. Ils nourrissent un imaginaire des vacances et du voyage (ordinaire ou scientifique). Mais ce voyage a la particularité de se dérouler « chez soi » sur place. En stimulant un voyage intérieur, un voyage en intérieur, ils se vivent, se ressentent plus qu'ils ne se disent ou ne se racontent. Les coquillages (trans)portent l'âme de ceux qui les ont recueillis, font rêver les enfants autant que les adultes, inspirent l'artiste. Ils font se croiser des registres qui a priori s'opposent: le proche et le lointain, le familier et l'exotique ou encore le commun et le scientifique. Leur présence apparaît suffisamment nécessaire pour que s'opère, d'une génération à l'autre, la transmission des valeurs qu'ils portent. Ils deviennent le symbole des migrations individuelles et/ou familiales. Mais plus que tout ils sont le point d'ancrage de la mémoire de lieux, de moments et de gens. Ils apparaissent comme des ponctuations qui suivent nos parcours de vie, qui en permettent les réminiscences. C'est en procédant à une auto-ethnographie élargie que l'auteur saisit le sens contemporain des territoires ordinaires des coquillages, inscrivant sa réflexion dans une ethnologie de la culture matérielle.

\section{ABSTRACT}

Ordinaries seashells at home or an invitation to a journey within. This article focuses on all sorts of shells that are in our homes. It questions the meaning of such shells. Shells combine both an aesthetic and mnemonic function. They have the particularity to surreptitiously creep into our lives. They accumulate over time here and there in the rooms of our homes, that it seems difficult to get rid of, which gives them a bit of eternity. They live in our homes as we live in them in return. They feed on an imaginary vacation and travel (ordinary or scientific). But this trip has the particularity to take place at home. By stimulating an inner journey, a journey inside, we live with and we feel them more than we can talk about them. Shells carry on the souls of those who have collected them, they make the children and the adults dreamt about them, and they inspire the artist. They make some opposite categories interact: the near and the far, the familiar and the exotic or the common and scientific. Their presence is considered as necessary enough for the values that they carry on, to be transmitted from one generation to another. They become the symbol of individual or familial migrations. But most of all they are the anchoring point of the memory places, times and people. They appear as pits on our life paths, which allow reminiscences. It is by making an extended auto-ethnography that the author captures the contemporary sense of the ordinary territories of the shells, inscribing her reflection in an anthropology of material culture.

\section{MOTS CLÉS}

Coquillage, décoration, espace domestique, souvenirs, transmission familiale, voyage

\section{KEYWORDS}

Seashell, decoration, domestic space, souvenirs, family transmission, travel 